\title{
Evaluation of hemolysis during storage of red blood cell concentrates processed by centrifugation and settling method by simple gravity in Burkina Faso
}

\begin{abstract}
Background: Hemolysis is one of the red blood cell concentrates (RCCs) storage concerns. In Burkina Faso, hemolysis rate was not routinely assessed in RCCs. Our study aimed at assessing the degree of hemolysis in RCCs processed by centrifugation (centrifuged RCCs) and whole blood settling by simple gravity (Sedimented RCCs) in Burkina Faso.

Methods: We conducted a cross-sectional comparative study on 46 prepared by centrifugation and 46 prepared by sedimentation, matched on their collection date and initial volume of whole blood. The hemolysis percent was measured on Days $0,7,14,21$, 28 and 32 .

Results: In the centrifuged RCCs, the hemolysis percent on D0 was $0.232 \%$ versus $0.199 \%$ for the sedimented RCCs $(\mathrm{p}=0.046)$. At D32, the average hemolysis percent was $0.835 \%$ for the sedimented RCCs and $0.779 \%$ for the centrifuged RCCs $(p=0.042)$. The degree of hemolysis increased gradually between D0 and D32 with an average increase of $0.120 \%$ for centrifuged RCCs and $0.116 \%$ for sedimented RCCs.

Conclusion: The degree of hemolysis at D32 in both centrifuged RCCs and sedimented RCCs falls below standards. Therefore, it necessary to revisit blood components processing procedures, focusing on the centrifugation parameters and the handling conditions.
\end{abstract}

Volume 9 Issue 3 - 202 I

\author{
Salam Sawadogo, ${ }^{1,2}$ Aboudou Moindze,' \\ Koumpingnin Nebie, ${ }^{1,2}$ Amadou Kafando, ${ }^{2}$ \\ Abdoul-Guaniyi Sawadogo, ${ }^{2}$ Patrice Ouili, ${ }^{2}$ \\ Eléonore Kafandol,3 \\ 'University Joseph KI-ZERBO, 03 BP 702I Ouagadougou 03, \\ Burkina Faso \\ ${ }^{2}$ National Blood Transfusion Center, 0I BP 5372 Ouagadougou \\ 0I, Burkina Faso \\ ${ }^{3}$ Charles De Gaulle Pediatric University Hospital, 979 Boulevard \\ des Tensoba, Ouagadougou, Burkina Faso
}

\begin{abstract}
Correspondence: Salam Sawadogo, University Joseph KIZERBO, 03 BP 702I Ouagadougou 03, Burkina Faso, Tel +22670032776, Email salemserein@hotmail.com, salam.sawadogo@ujkz.bf
\end{abstract}

Received: May 23, 202I | Published: June 30, 2021

Keywords: red cell concentrate, hemolysis, storage lesions, centrifugation, storage duration

Abbreviations: RCC, Red cell concentrate; RBC, Red blood cell; Hb, Hemoglobin; USA, United State of America; \%, percent; $\mathrm{RBTC}$, regional blood transfusion center; CPD, citrate-phosphatedextrose; SAGM, saline-adenine-glucose-mannitol solution; G6PD, glucose-6-phosphate deshydrogenase; NBTC, national blood transfusion center; CIRS, internal scientific review committee; $\mathrm{cm}$, centimeter; D, day; $\mathrm{mL}$, milliliter; $\mathrm{S}$ RCC, sedimented red cell concentrate; $\mathrm{C}$ RCC, centrifuged $\mathrm{r} 4 \mathrm{ed}$ cell concentrate; ${ }^{\circ} \mathrm{C}$, degree celsius; g, gravitation symbol

\section{Introduction}

Red blood cells (RBCs) undergo multiple changes during storage known as storage lesions. Indeed, they change shapes, from the normal discoid form to echinocytes and finally to spherical form, become more rigid, shed lipid, exhibit fall in Adenosine-triphosphate and 2,3-diphosphoglycerate content before the membrane ruptures. ${ }^{1}$ This rupture causes the release of free hemoglobin $(\mathrm{Hb})$ into the suspending fluid. At visual inspection, hemolysis appears as an orange-red color, due to the presence of $\mathrm{Hb}$ in the supernatant..$^{2-5}$

RBCs hemolysis can occur either during blood components processing or handling, transportation and storage. ${ }^{2-5}$

It is the consequence of several factors comprising blood components processing procedures (time between blood collection and separation, the type of anticoagulant and preservative solution in the blood bag, the centrifugation speed), handling and storage conditions, RBCs intrinsic abnormalities, blood donors characteristics. ${ }^{4,6,7}$
As one main indicator of the amount of $\mathrm{RBC}$ storage lesions, hemolysis in stored red cell concentrate (RCC) units raises many issues. Firstly, it can affect the dose of oxygen-carrying capacity (ie $\mathrm{Hb}$ within erythrocytes) that the blood unit must provide if transfused. Secondly, given the toxicity of free $\mathrm{Hb}$, harmful consequences, such as kidney damage could occur if several RCC units with significant hemolysis were transfused to the patient. ${ }^{8}$

Several techniques are used to assess the measurement of hemolysis in RCCs, with the tetramethylbenzidine method, based on spectrophotometry that is considered as the "gold standard". Other techniques use photometry or microplate methods. ${ }^{9-11}$ But these tests are not well suited to a blood manufacturing and delivery settings. They are cumbersome and expensive. The most common technique used in blood services is the visual inspection. Before delivery, the blood unit is inspected for a hemolytic supernatant. ${ }^{5}$ However, this qualitative method has limitations even with colorimetric scales as a visual comparator for estimating hemolysis levels. ${ }^{12,13}$

It also lacks adequate standardization making inter-laboratory comparison difficult. ${ }^{9}$ So, according, to the experience of the health worker who inspects the blood unit, the degree of hemolysis could be either overestimated, leading to unjustified destruction of RCC units, or underestimated, with the risk of transfusion of RCC units which contain high levels of free $\mathrm{Hb}$.

In this context, blood services must implement quality systems with proven procedures to control blood components manufacturing, handling and storage processes. Such a quality system must include 
quality controls on ready-to-use blood products with at-end-storage hemolysis thresholds not to be exceeded. These thresholds are less than $1 \%$ in USA and $0.8 \%$ in Europe.$^{3,14}$ Recently, the US Food and Drug Administration added the "95/95 rule" which requires that $95 \%$ of units meet the standard with $95 \%$ statistical certainty. ${ }^{3}$ Some studies showed degree of hemolysis between 0.2 and $0.4 \%$ and 96.7 to $99.5 \%$ of units with an hemolysis percent less than $1 \%^{3,14}$ at day 42 .

In Burkina Faso, RCCs are processed from whole blood using the classic centrifugation technique. However, in some Regional Blood Transfusion Centers (RBTCs) without suitable facilities for centrifugation, the alternative settling method by simple gravity as previously described.$^{15}$ was used. The quality control on ready-to-use RCCs performed in RBTCs was limited to the measurement of the unit volume and the $\mathrm{Hb}$ content (i.e the entire amount of $\mathrm{Hb}$ in the blood unit). Changes occurring during the storage, mainly hemolysis, were not covered. ${ }^{16,17}$

Our study aimed at assessing the degree of hemolysis in RCC units processed both by centrifugation and settling methods in Burkina Faso.

\section{Material and methods}

\section{Study setting}

We conducted a cross-sectional comparative study at the RBTCs of Ouagadougou (RBTC-O) and Koudougou (RBTC-K). These RBTCs represent respectively the biggest and the third blood centers in Burkina Faso and collect annually around 40,000 and 12,000 blood units.

Burkina Faso is a low-income country that experiencing blood shortage and poor quality and unsafe blood transfusions. ${ }^{18} \mathrm{~T}$ ropical infections such as malaria, dengue fever and others transfusion transmitted diseases are endemic. Blood products are issued mainly for anemia resulting from malaria, genetic red blood cell abnormalities and obstetrical hemorrhages ${ }^{19}$ Blood is collected from 18-60 years' voluntary unpaid blood donors of both genders. Each donor underwent a pre-donation interview conducted by trained healthcare workers using standardized selection questionnaire. Beside the donors' behaviors and other viral infectious risk factors, signs of acute infections as malaria or bacterial infections and chronic diseases comprising hemoglobinopathies, chronic inflammations are assessed..$^{20}$ Blood is collected in triple bags system, the main bag containing $63 \mathrm{~mL}$ of CPD-anticoagulant (citrate, phosphate, dextrose) and one of the two satellite bags containing $100 \mathrm{~mL}$ of SAGM additive preservative solution (saline, adenine, glucose, mannitol). Units of less than $300 \mathrm{~mL}$ or more than $510 \mathrm{~mL}$ are not suitable for therapeutic use. ${ }^{16}$ Apart from the screening of serological markers for human immunodeficiency virus infection, hepatitis $\mathrm{B}$ and $\mathrm{C}$ and syphilis as well as the pre-donation $\mathrm{Hb}$ measurement, no other biological analysis is performed on the collected blood units. ${ }^{18}$
At the RBTC-O, whole blood is typically processed within 48 hours of collection into lecukocyte containing RCCs. They are centrifuged at $2490 \mathrm{~g}$ for 20 minutes and then the plasma is extracted using manual presses. Less than $15 \%$ of whole blood are processed into platelet concentrates and fresh frozen plasma. In the RBTC-K, the whole blood bags are stored vertically at a temperature of $4 \pm 2$ ${ }^{\circ} \mathrm{C}$ for at least 72 hours, then the plasma is extracted and destroyed. ${ }^{15}$ The centrifuged RCCs (C_RCCs) and the sedimented ones (S_RCCs) are re-suspended in $100 \mathrm{~mL}$ SAGM additive preservative solution and stored in temperature-controlled refrigerators between $2-6{ }^{\circ} \mathrm{C}$ for maximum 42 days. Upon the blood unit delivery, it is visually inspected for hemolysis signs or other macroscopic abnormalities.

\section{Study materials}

Selection of RCC units: Our study randomly included 92 ready-touse RCCs comprising 46 units of C_RCCs and 46 units of S_RCCs, matched each other on the date of collection and the initial volume of whole blood $\pm 50 \mathrm{~mL}$. The units that presented macroscopic abnormalities on visual inspection (presence of clots, loss of the bag integrity, greenish or blackish coloration) were not included. The RCCs included in the study were kept under standard storage conditions. ${ }^{16}$ The day of blood collection was considered as day 0 (D0). On D0, D7, D14, D21, D28 and D32, the RCCs were visually inspected and blood samples taken (as described below) for the measurement of supernatant hemoglobin (supernatant $\mathrm{Hb}$ ).

Blood sampling and biological analyzes: A blood sampling method that preserved RCC unit for delivery to patients who needed blood transfusion at the end of study, was carried out. After blood collection and processing, a total length of $100-120$ centimeters $(\mathrm{cm})$ of the bag tubings (tubing connecting the main bag to the needle and to the satellite bags) was kept for quality controls and compatibility tests (before delivery) purposes. These tubings are stripped and clamped in order to mix tubing blood with the blood in the bag. The bag is homogenized by successive inversions and then the tubing is refilled and $10-15 \mathrm{~cm}$ is sealed and cut for blood sampling in an anticoagulant-free tube. Blood samples was collected by this way on D0, D7, D14, D21, D28 and D32. These samples were centrifuged for 5 minutes at $2500 \mathrm{rpm}$ and the supernatant was used for supernatant $\mathrm{Hb}$ measurement.

For each RCC unit, the total hemoglobin (Total $\mathrm{Hb}$ ) and hematocrit were measured at D0 using an ABX MICROS 60 ES hematology analyzer (HORIBA ABX SAS, Kyoto, Japan). The supernatant $\mathrm{Hb}$ was measured at D0, D7, D14, D21, D28 and D32 on a spectrophotometer UV-Visible Cecil Aquarius CE 7400 (Cecil Instruments Limited, Cambridge CB24 6AZ, Royaume-Uni) using the photometric method with determination at end point at a wavelength of $540 \mathrm{~nm}$. The degree of hemolysis expressed in percentage of free $\mathrm{Hb}$ in relation to the total $\mathrm{Hb}$ (i.e. free and intraerythrocytic $\mathrm{Hb}$ ) of the unit was calculated at each measurement using this formula:

$$
\text { Hemolysis percent }(\%)=\frac{\left[(100-\text { Hematocrit }) \times \text { Supernatant } H b\left(g . d l^{-1}\right)\right]}{\text { Total } H b\left(g \cdot d l^{-1}\right)}
$$

\section{Data management and statistical analysis}

Data was entered using Epi-Data 3.1 and analyzed using Stata 15 The minimum, mean and maximum values of the hemolysis percent were calculated for each type of RCCs at D0, D7, D14, D21, D28 and D32. The average hemolysis percent for each type of RCCs from D7 to D32 was compared to D0 hemolysis using paired t-test. The average variation in hemolysis between D0 and D32 for C_RCCs and S_RCCs were compared using a paired t test at a significant level of $\mathrm{p}<0.05$.

\section{Ethical considerations}

The study protocol was approved by the Internal scientific review committee (CIRS) of the National blood transfusion center (NBTC). 
Whole blood was collected from voluntary non-remunerated blood donors. All have signed an informed consent so that the collected blood and samples undergo mandatory analyzes and be used for research. The study was conducted with respect to the quality system requirements of the NBTC. So, we carried out a blood sampling method that preserved the integrity and quality of the RCC units. RCC units with macroscopic hemolysis or a hemolysis rate greater than $0.8 \%$ were discarded.

\section{Results}

The characteristics of the 92 RCCs included in our study are stated in Table 1. At baseline, there were difference in volume, $\mathrm{Hb}$ content and hematocrit between the centrifuged RCCs and sedimented ones. The hemolysis percent at D0 was $0.232 \%$ and $0.199 \%$ respectively for C_RCCs and S_RCCs $(\mathrm{p}=0.04)$. The maximum hemolysis was observed on D32 for the two groups, with $1.460 \%$ for C RCCs versus
$1.137 \%$ for S_RCCs. A hemolysis percent greater than $1 \%$ were observed in C_RCCs on D14 and on D28 for S_RCCs (Table 2).

Table I Overview of the baseline characteristics of the 92 red cell concentrates included in the study Burkina Faso

\begin{tabular}{|c|c|c|c|}
\hline Parameter & $\begin{array}{l}\text { Centrifuged } \\
\text { RCC (mean } \\
\pm \text { sd) }\end{array}$ & $\begin{array}{l}\text { Sedimented } \\
\text { RCC (mean } \\
\pm \text { sd) }\end{array}$ & $\begin{array}{l}\text { Reference } \\
\text { values }\end{array}$ \\
\hline Volume $(\mathbf{m L})^{\mathrm{p}}$ & $240.4 \pm 35.7$ & $320.30 \pm 48.9$ & $130-380$ \\
\hline $\begin{array}{l}\text { Hemoglobin } \\
\text { content }(g)^{p}\end{array}$ & $55.1 \pm 8.5$ & $49.3 \pm 7.6$ & $>40$ \\
\hline Hematocrit (\%) ${ }^{\mathrm{p}}$ & $56.2 \pm 4.5$ & $48.2 \pm 6.3$ & $50-70$ \\
\hline
\end{tabular}

RCC, red cell concentrates; $\mathrm{mL}$, milliliter; g, gram; \%, percentage; sd, standard deviation; $\mathrm{P}: \mathrm{p}<0.05$

Table 2 Mean, minimum and maximum hemolysis percent in centrifuged and sedimented red cell concentrates according to storage duration, Burkina Faso

\begin{tabular}{llllllll}
\hline \multirow{2}{*}{$\begin{array}{l}\text { Storage } \\
\text { duration }\end{array}$} & \multicolumn{2}{l}{ Centrifuged RCC } & \multicolumn{2}{l}{ Sedimented RCC } & p-value \\
\cline { 2 - 7 } & Min (\%) & Average (\%) & Max (\%) & Min (\%) & Average (\%) & Max (\%) & \\
\hline D0 & 0.108 & 0.232 & 0.523 & 0.098 & 0.199 & 0.307 & 0.046 \\
D7 & 0.158 & 0.383 & 0.784 & 0.104 & 0.234 & 0.488 & $<0.001$ \\
D14 & 0.163 & 0.489 & 1.121 & 0.148 & 0.368 & 0.605 & $<0.001$ \\
D21 & 0.176 & 0.534 & 1.269 & 0.197 & 0.525 & 0.875 & 0.125 \\
D28 & 0.226 & 0.677 & 1.181 & 0.227 & 0.619 & 1.099 & 0.035 \\
D32 & 0.285 & 0.835 & 1.460 & 0.380 & 0.779 & 1.137 & 0.042 \\
\hline
\end{tabular}

D, day; RCC, red cell concentrates; Min, minimum; Max, maximum; \%, percentage

At D32, the average hemolysis percent was almost equal to $0.8 \%$ for the S RCCs and greater than $0.8 \%$ for C RCCs. We noted that $16.7 \%(7 / 42)$ of C_RCCs and 7.1\% (3/42) of S_RCCs had a hemolysis percent greater than $0.8 \%$ at D32. Two C RCCs and one S RCC presented a macroscopic hemolysis detected at visual inspection at D32.
The hemolysis percent increased gradually from D0 to D32 (Figure 1) with an average increase of $0.120 \%$ and $0.116 \%$ between two measurements respectively for C_RCCs and S_RCCs. A total average increase of $0.603 \%$ and $0.580 \%$ was noted respectively for C_RCCs and S_RCCs $(\mathrm{p}=0.02)$.

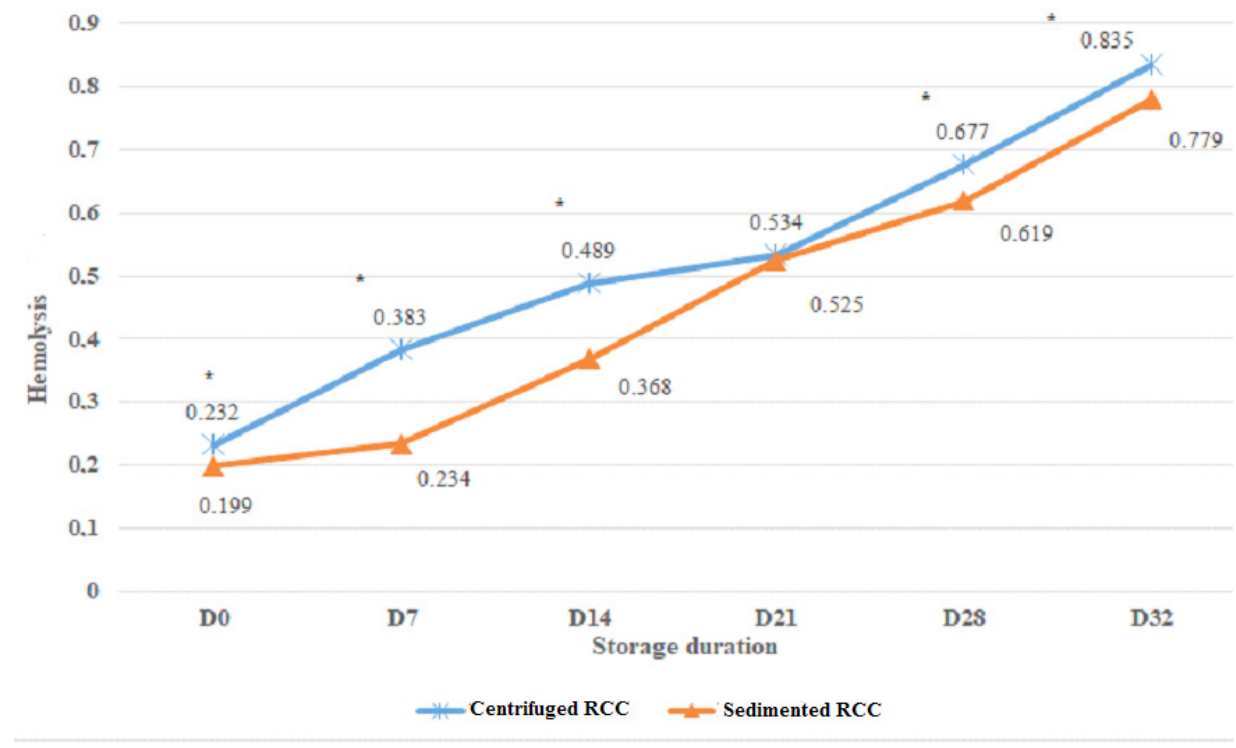

*: $p<0.05$ (Paired t-test)

Figure I Evolution of degree of hemolysis in centrifuged and sedimented red cell concentrates during storage, Burkina Faso. 


\section{Discussion}

Our study aimed at assessing the degree of hemolysis in RCCs units prepared by two methods, centrifugation and sedimentation. We found that the hemolysis percent increased gradually between D0 and D32 with an average increase of $0.120 \%$ for centrifuged RCCs and $0.116 \%$ for sedimented RCCs. At D32, the average hemolysis percent was $0.835 \%$ for the sedimented RCCs and $0.779 \%$ for the centrifuged $\operatorname{RCCs}(p=0.042)$.

These findings give actual information on an aspect of the quality of RCCs that is not took into account in routine quality controls. Indeed, the quality control on the ready-to-use blood components in Burkina Faso is limited to the control of volume and hemoglobin content. However, the document on the standard and characteristics of blood components in Burkina Faso ${ }^{16}$ as well as the standards for the steps-by-steps accreditation program of the Africa Society of Blood Transfusion ${ }^{21}$ prescribe the control of hemolysis rate. However, our study has some limitations. We did not assess that at-end-storage (at D42) hemolysis levels; patient demand for blood was not fully covered and it would not be ethical to store blood units longer than 32 days just for our study purposes. Our study assessed only the hemolysis percent that is certainly an important parameter of the RBCs storage lesions, but it is not the only one. The dosage of potassium and lactate dehydrogenases in plasma could be informative. Moreover, since we have not tested the presence of plasmodium or other hematophagous parasites in the blood units as well as any abnormal hemoglobin such as $\mathrm{Hb} \mathrm{S}$ or $\mathrm{C}$ (which are frequent in our context), we were therefore unable to analyze their influence on the degree of hemolysis of the RCCs.

The D32 hemolysis percent $(0.835 \%$ and $0.779 \%$ respectively for C RCCs and S RCCs) was higher than those noted by other authors at D42. Indeed, Makroo et al. ${ }^{2}$ and Arif et al. ${ }^{1}$ in India noted at-end-storage hemolysis percent of $0.553 \%$ and $0.359 \%$ respectively in RCCs containing a SAGM additive solution. Gkoumassi et al. ${ }^{3}$ in the Netherlands found at-end-storage hemolysis percent varying from $0.267 \%$ to $0.304 \%$. Several factors can explain these differences including inappropriate handling during processing, improper storage conditions, high centrifugation speed, rapid addition of additive preservative solution, variation in the quality of blood bags, bacterial hemolysins, antibodies causing complement mediated lysis, red cell membrane defects.

The studies in India and Netherlands included leukoreduced SAGM RCCs, contrary to our context where leukoreduction was not practiced. Leukocytes contain numerous chemical components and enzymes like proteases, which are involved in RBCs hemolysis. Also, leukoreduction halves the occurrence of hemolysis as showed by Hess et al., with a reduction of the hemolysis percent at D43 from $0.34 \pm 0.29 \%$ to $0.16 \pm 0.07 \%$ on more than 10,000 RCCs. ${ }^{14}$ In another study, authors noted that all the RCC units with more than $1 \%$ of hemolysis concerned non-leukoreduced units. ${ }^{22}$

Furthermore, the centrifugation speeds can induce a physical stress on RBCs and predisposes them to hemolysis. In our study, C RCCs were obtained after a centrifugation at $2490 \mathrm{~g}$ for 20 minutes (approximately $2225 \mathrm{rpm}$ ), unlike the study by Arif et al. ${ }^{1}$ where the whole blood units were centrifuged at $1750 \mathrm{rpm}$ for 9 minutes. We found a significant difference in hemolysis percent between RCCs obtained by centrifugation and decantation, supporting the hypothesis of an impact of the centrifugal force.

In addition, in our context, the storage period before transformation was quite long (48 - 72 hours) while it was 24 hours maximum in most of the referred studies. Such long delays favor the degradation of leukocytes and the release of their toxic substances that alter RBC membrane. This long storage time before transformation and the hard centrifugation in our context probably explain the very high hemolysis percent at D0 $(0.232 \%$ for C RCCs and $0.199 \%$ for S RCCS). In similar studies, the hemolysis at D0 was $0.027-0.057 \%$ in the Netherlands ${ }^{3}$ and $0.81-0.1 \%$ in India. ${ }^{1,2}$ The hemolysis percent at D0 in our study was sometimes higher than the at-end-storage (D42) hemolysis percent in some studies in Europe. ${ }^{3,14}$ This testifies to the potential deleterious effects of blood collection kits, blood components processing techniques and equipment and blood storage conditions on RBCs.

Beside the impact of the factors cited above, we can discuss certain characteristics of blood donors. Indeed, the correlation between RBC defects (membrane, hemoglobin and enzymes abnormalities) and the occurrence of hemolysis is well described. RBCs of individuals with G6PD deficiency, hemoglobinopathies may be more prone to hemolysis. ${ }^{23}$ Physiological differences related to gender could have some impact. Indeed, RCCs from male donors were more likely to hemolyze. ${ }^{24,25}$ Female blood on average hemolyzed $21 \%$ less than male blood. The difference seems to be greatest during donor's reproductive years, suggesting a possible role of sex hormones. ${ }^{25}$

The hemolysis percent gradually increased from D0 to D32, both in centrifuged RCCs $(+0.603 \%)$ and in sedimented ones $(+0.580)$ with respectively an average increase between two measures of $0.120 \%$ and $0.116 \%$. This observation is expected and was already underlined. ${ }^{1-4,23}$ It reflects the gradually cellular changes occurring during storage. $4,6,26$

We had hemolysis percent greater than $0.8 \%$ in centrifuged RCCs and sedimented RCCs respectively at D14 and D18. At D32, there were $16.7 \%$ of centrifuged RCCs and $7.1 \%$ of sedimented RCCs had hemolysis percent greater than $0.8 \%$. In the Indian studies, such an achievement was not observed. ${ }^{1,2,27}$ These findings show that the RCCs produced in Burkina Faso, mainly by centrifugation, do not fulfill standards requirements that fix the hemolysis threshold at less $0.8 \%{ }^{16}$ The evolution of hemolysis percent on Figure 1 clearly suggests that at the end of storage on D42, the hemolysis percent will be far beyond the authorized limit, both for centrifuged and sedimented RCCs. This is a major concern, since visual inspection seems to have a low sensitivity to detect hemolysis. ${ }^{5}$ In our study, the visual inspection have detected hemolysis only in three units. This questions the safety blood units delivered after visual inspection and transfused to patients in many blood transfusion services. These cases could cause adverse reactions in patients due to the harmfulness of free hemoglobin on certain organs. Circulating free hemoglobin can bind with nitric oxide at endothelium and induce vasoconstriction, endothelial dysfunction and platelet activation, which increases the risk for thrombus formation. ${ }^{27}$ It can also cause kidney damage. ${ }^{8}$ Unfortunately, because of weakness of hemovigilance system in subsaharan countries, these adverse reactions are underreported. ${ }^{19}$

We noted that hemolysis percent was significantly higher in C RCCs than in S RCCs from D0 to D14 and D28. In addition to the physical stress due to centrifugation already mentioned above, we can discuss the volume of residual plasma. Indeed, as shown on table I, the volume of sedimented RCCs was greater than the volume of centrifuged RCCs, due to a larger volume of residual plasma in sedimented RCCs ( $81 \mathrm{~mL}$ versus $25 \mathrm{~mL}$ ) reported in previous study. ${ }^{15}$ So, sedimented RCCs could build up lesser levels of lactate and metabolic waste products that might affect hemolysis late in storage. 


\section{Conclusion}

The RCCs during their preparation and storage undergo hemolysis. Our study shows that the percent hemolysis for both centrifuged and sedimented RCCs observed in Burkina Faso fall below the international standards. So, the National blood transfusion service in Burkina Faso needs to review and improve RCC processing methods. The major points to revisit are the storage and handling conditions, mainly the time from whole blood collection to blood components processing that must be reduced at most 24 hours, the centrifugation parameters.

\section{Acknowledgments}

Thanks to all the staff of the NBTC.

\section{Conflicts of interest}

None.

\section{References}

1. Arif SH, Yadav N, Rehman S, et al. Study of Hemolysis During Storage of Blood in the Blood Bank of a Tertiary Health Care Centre. Indian $J$ Hematol Blood Transfus. 2017;33(4):598-602.

2. Makroo R, Raina V, Bhatia A, et al. Evaluation of the red cell hemolysis in packed red cells during processing and storage. Asian J Transfus Sci. 2011;5:15.

3. Gkoumassi E, Dijkstra-Tiekstra MJ, Hoentjen D, et al. Hemolysis of red blood cells during processing and storage:HEMOLYSIS DURING PROCESSING AND STORAGE. Transfusion (Paris). 2012;52:489-492.

4. Sowemimo-Coker SO. Red blood cell hemolysis during processing. Transfus Med Rev 2002;16(1):46-60.

5. Janatpour KA, Paglieroni TG, Crocker VL, et al. Visual assessment of hemolysis in red blood cell units and segments can be deceptive. Transfusion (Paris) 2004;44(7):984-989.

6. Hess JR. Red cell changes during storage. Transfus Apher Sci 2010;43(1):51-59.

7. Orlov D, Karkouti K. The pathophysiology and consequences of red blood cell storage. Anaesthesia. 2015;70:29-e12.

8. Buehler PW, D'Agnillo F. Toxicological Consequences of Extracellular Hemoglobin: Biochemical and Physiological Perspectives. Antioxid Redox Signal 2010;12(2):275-291.

9. Cookson P, Sutherland J, Cardigan R. A simple spectrophotometric method for the quantification of residual haemoglobin in platelet concentrates. Vox Sang. 2004;87(4):264-271.

10. Seghatchian J, Krailadsiri P, Beard M, et al. Development of a microplate method for the measurement of plasma haemoglobin and its use in monitoring the storage lesion of red cell components. Transfus Apher Sci. 2002;26(1):91-93.

11. Zimmermann $\mathrm{R}$, Heidenreich $\mathrm{D}$, Weisbach $\mathrm{V}$, et al. In vitro quality control of red blood cell concentrates outdated in clinical practice. Transfus Clin Biol 2003;10:275-283.

12. Lippi G, Plebani M, Di Somma S, et al. Hemolyzed specimens: a major challenge for emergency departments and clinical laboratories. Crit Rev Clin Lab Sci. 2011;48(3):143-153.
13. Luksic AH, Nikolac Gabaj N, Miler M, et al. Visual assessment of hemolysis affects patient safety. Clin Chem Lab Med CCLM 2018;56(4):574-581.

14. Hess JR, Sparrow RL, Van Der Meer PF, et al. Red blood cell hemolysis during blood bank storage: using national quality management data to answer basic scientific questions: RBC hemolysis during storage. Transfusion (Paris). 2009;49(12):2599-2603.

15. Sawadogo S, Nébié K, Kafando E, et al. Preparation of red cell concentrates in low-income countries: Efficacy of whole blood settling method by simple gravity in Burkina Faso. Int $J$ Blood Transfus Immunohematol. 2016;6:20-29.

16. Centre national de transfusion sanguine. Normes et caractéristiques des produits sanguins labiles au Burkina Faso. Version 2, Ouagadougou: Ministère de la santé, 2016.

17. Nebie K, Sawadogo S, Kafando E, et al. Role of quality control for improvement of blood components in the Regional Blood Centre of Ouagadougou in Burkina Faso, 2014. Transfus Clin Biol. 2017;24:431439.

18. Nébié K, Ouattara S, Sanou M, et al. Poor procedures and quality control among nonaffiliated blood centers in Burkina Faso:an argument for expanding the reach of the national blood transfusion center: blood center management in Burkina Faso. Transfusion (Paris) 2011;51(7pt 2):16131618 .

19. Sawadogo S, Nebie K, Millogo T, et al. Traceability of Blood Transfusions and Reporting of Adverse Reactions in Developing Countries:A Six-Year Postpilot Phase Experience in Burkina Faso. Adv Hematol. 2018;2018:1-

20. Ministère de la santé. Arrêté portant directives nationales de Bonnes pratiques transfusionnelles au Burkina Faso. N²014-589/MS, 2014.

21. Comité des Normes de la SATS (C Tayou Tagny). Référentiel de la SATS Programme d'Accréditation par Etapes.

22. McAteer MJ, Dumont LJ, Cancelas J, et al. Multi-institutional randomized control study of haemolysis in stored red cell units prepared manually or by an automated system: Potential association between whole blood donor and haemolysis. Vox Sang. 2010;99:34-43.

23. Kouamenan S, Sékongo Y, Konan S, et al. Etude de la cinétique de l'hémolyse dans les poches de concentrés érythrocytaires des donneurs de sang hétérozygotes AS et son implication transfusionnelle. Afr Sang 2016;18:4-7.

24. Jordan A, Chen D, Yi Q-L, et al. Assessing the influence of component processing and donor characteristics on quality of red cell concentrates using quality control data. Vox Sang. 2016;111(1):8-15.

25. Kanias T, Sinchar D, Osei-Hwedieh D, et al. Testosterone-dependent sex differences in red blood cell hemolysis in storage, stress, and disease: testosterone and sex differences in hemolysis. Transfusion (Paris). 2016;569(10):2571-2583

26. Hess JR. An update on solutions for red cell storage. Vox Sang. 2006;91(1):13-19.

27. Saini N, Basu S, Kaur R, et al. Assessment of changes in plasma hemoglobin and potassium levels in red cell units during processing and storage. Transfus Apher Sci 2015;52(3):319-325. 